\title{
33. GRANITOPHILE TRACE ELEMENTS AND ALTERATION IN BASALTS AND SERPENTINITES FROM HOLES 332B AND 334, LEG 37 DSDP
}

D.M. Shaw and J.R. Muysson, Department of Geology, McMaster University, Hamilton, Ontario, Canada

\begin{abstract}
Preliminary study of 26 basalts and 2 serpentinites from Hole 332B and Site 334 shows typical ocean-floor basalt abundances of $\mathrm{Li}, \mathrm{Rb}, \mathrm{Sr}, \mathrm{Ba}$, and $\mathrm{Tl}$ and no correlation with depth below the sea floor.
\end{abstract}

\section{INTRODUCTION}

The purpose of this study is to follow the geochemical effects of alteration on the behavior of $\mathrm{Li}, \mathrm{Rb}$, $\mathrm{Sr}, \mathrm{Ba}$, and $\mathrm{Tl}$, in the basalts and other rocks: the relationship with depth could be important in this connection, since it is uncertain how a spreading ridge grows.

\section{METHODS}

The powders supplied from Dalhousie University were analyzed by AAS (Table 1): in the case of $\mathrm{Tl}$ a chemical pre-enrichment was made following the method of Fratta (1974). Table 1 includes analyses of the standard reference rocks W-1 and BR; comparison with Flanagan's (1973) compilation suggests that our results are satisfactory.

Study of thin sections permitted a rough classification of the degree of alteration, in terms of feldspar, olivine, pyroxene and groundmass alteration, presence of chlorite, smectite, etc. (Table 2.)

\section{RESULTS}

Table 3 shows the mean contents of $\mathrm{Li}, \mathrm{Rb}, \mathrm{Sr}, \mathrm{Ba}$ for the 26 Leg 37 basalts analyzed so far, compared with mean values determined at McMaster University (mostly unpublished, but see Shaw et al., 1974, a, b). As expected the element abundances are close to other oceanfloor basalts and show no sign of the $\mathrm{Li}$ enrichment characteristic of French spilitic rocks (Vatin-Pérignon and Shaw, 1972).

The relationship between LOI and degree of alteration is shown in Table 2. Comparison is impeded by the fact that the basalts are almost all vesicular and the vesicles are filled with smectite, carbonate, and often zeolites. Volatile components in these minerals contribute to the LOI, which therefore is not only a function of alteration, Nevertheless there is clearly no simple relationship between depth in the drill hole and degree of alteration (except for the two serpentinites). The same conclusion applies when the trace element analyses in Table 1 are examined.

If a spreading ridge is an environment of convective hydrothermal circulation as first proposed by Y. Bottinga and recently discussed by Spooner and Fyfe (1973), it is clear that alteration of such intensity must lie below the levels penetrated by the Leg 37 drilling program.

\section{ACKNOWLEDGMENTS}

Financial assistance from the National Research Council of Canada through Grants A0155 and DAG-9 is gratefully acknowledged.

\section{REFERENCES}

Flanagan, F.J., 1973. 1972 values for international geochemical reference samples: Geochim. Cosmochim. Acta, v. 37 , p. $1189-1200$.

Fratta, M., 1974. AAS determination of ppb amounts of T1 in silicate rocks: Canadian J. Spectroscopy, v. 19, p. 3337.

Shaw, D.M., Dupuy, C., Fratta, M. and Helsen, J., 1974a. An application of factor analysis to basic volcanic rock geochemistry: Bull. Volcanol., v. 38, p. 1070-1089.

Shaw, D.M., Fratta, M., Dupuy, C. and Vatin-Pérignon, N., 1974b. Comportement de quelques éléments incompatibles dans les roches volcanologiques à caractère tholéiitique, alcalin et spilitique: Rev. Haute Auvergne, v. 44, p. $415-440$.

Spooner, E.T.C. and Fyfe, W.S., 1973. Sub-sea-floor metamorphism, heat and mass transfer: Contrib. Mineral. Petrol., v. 42, p. 287-304.

Vatin-Pérignon, V. and Shaw, D.M., 1972. Comportement du $\mathrm{Li}$ et d'autres éléments en traces dans quelques coulées triasiques de l'Aiguille de Morges: Géol. Alpine, v. 48, p. 201-206. 
TABLE 1

Analysis Results up to July 1975 in $\mathrm{ppm}$ except $\mathrm{Tl}(\mathrm{ppb})^{\mathrm{a}}$

\begin{tabular}{|c|c|c|c|c|c|c|c|}
\hline $\begin{array}{c}\text { Sample } \\
\text { Interval in } \mathrm{cm}\end{array}$ & $\mathrm{Li}$ & $\mathrm{Rb}$ & $\mathrm{Sr}$ & $\mathrm{Ba}$ & $\mathrm{Tl}$ & $\mathrm{Cr}$ & $\begin{array}{l}\text { Alteration } \\
\text { Index }\end{array}$ \\
\hline \multicolumn{8}{|l|}{ Hole 332B } \\
\hline $\begin{array}{l}2, \mathrm{CC}(1) \\
3-3,126-128(4) \\
4-1,100-102(1) \\
6-3,34-36(3) \\
8-3,93-95(11) \\
15-1,73-75(7) \\
16-1,141-143(19) \\
18-1,86-88(9) \\
23-1,15-17(2 \mathrm{~A}) \\
23-1,78-80(6 \mathrm{~A}) \\
25-2,95-97(13) \\
33-1,95-98(10 \mathrm{G}) \\
35-2,114-117(10) \\
35-3,61-64(1 \mathrm{H}) \\
36-4,99-101(6 \mathrm{~B}) \\
37-1,78-80(10) \\
37-3,28-29(3) \\
40-1,30-32(3 \mathrm{~B}) \\
42-1,56-58(3 \mathrm{D}) \\
44-5,68-70(6 \mathrm{~B}) \\
48-1,30-33(3) \\
48-1,118-120(9 \mathrm{C})\end{array}$ & $\begin{array}{c}7.0 \\
4.6 \\
10 \\
13 \\
5.6 \\
9.3 \\
18 . \\
15 \\
7.0 \\
3.0 \\
22 \\
7.0 \\
13 \\
6.5 \\
5.1 \\
7.0 \\
10 \\
7.9 \\
7.0 \\
9.3 \\
5.1 \\
4.6\end{array}$ & $\begin{array}{c}4.6 \\
0 \\
7.3 \\
8.2 \\
5.5 \\
2.7 \\
3.7 \\
3.7 \\
4.6 \\
6.4 \\
4.6 \\
1.8 \\
5.5 \\
7.3 \\
8.2 \\
7.3 \\
5.5 \\
9.1 \\
5.5 \\
11 \\
5.5 \\
5.5\end{array}$ & $\begin{array}{r}101 \\
97 \\
121 \\
130 \\
118 \\
118 \\
94 \\
83 \\
91 \\
100 \\
101 \\
132 \\
94 \\
90 \\
93 \\
110 \\
93 \\
127 \\
93 \\
118 \\
127 \\
118\end{array}$ & $\begin{array}{r}43 \\
37 \\
60 \\
64 \\
60 \\
53 \\
56 \\
39 \\
27 \\
42 \\
50 \\
61 \\
39 \\
47 \\
\mathrm{~b} \\
\mathrm{~b} \\
27 \\
\mathrm{~b} \\
27 \\
120 \\
54 \\
0\end{array}$ & $\begin{array}{r}25 \\
59 \\
13 \\
17 \\
15 \\
44 \\
16 \\
16 \\
37 \\
82 \\
19 \\
8 \\
\mathrm{~b} \\
50 \\
56 \\
24 \\
66 \\
47 \\
69 \\
29 \\
39 \\
46\end{array}$ & $\begin{array}{l}370 \\
380 \\
160 \\
300 \\
270 \\
270 \\
630 \\
820 \\
470 \\
420 \\
470 \\
150 \\
670 \\
860 \\
430 \\
370 \\
620 \\
270 \\
570 \\
190 \\
390 \\
430\end{array}$ & $\begin{array}{l}1 \\
1 \\
2 \\
2 \\
1 \\
1 \\
1 \\
1 \\
2 \\
2 \\
2 \\
3 \\
1 \\
3 \\
2 \\
4 \\
1 \\
3 \\
2 \\
4 \\
2 \\
4\end{array}$ \\
\hline \multicolumn{8}{|l|}{ Site 334} \\
\hline $\begin{array}{l}15-2,14-17(2 \mathrm{~A} \\
16-1,40-42(4) \\
19-3,99-105(12) \\
20-2,26-28(1 \mathrm{~B}) \\
22-2,80-82(6 \mathrm{~B}) \\
23-2,78-82(11) \\
\text { Standard rock W-1 }\end{array}$ & $\begin{array}{r}6.5 \\
7.0 \\
5.6 \\
14\end{array}$ & $\begin{array}{l}8.2 \\
2.7 \\
6.4 \\
5.5\end{array}$ & $\begin{array}{l}85 \\
93 \\
85 \\
85\end{array}$ & $\begin{array}{r}110 \\
45 \\
99 \\
72\end{array}$ & $\begin{array}{r}35 \\
\mathrm{~b} \\
44 \\
17 \\
\\
22\end{array}$ & $\begin{array}{l}230 \\
230 \\
210 \\
220\end{array}$ & $\begin{array}{l}1 \\
1 \\
1 \\
1 \\
4 \\
4\end{array}$ \\
\hline $\begin{array}{l}\text { Analysis } 75020 \\
\text { Analysis } 75062 \\
\text { Flanagan (1973) }\end{array}$ & $\begin{array}{l}12 \\
13 \\
14.5\end{array}$ & $\begin{array}{l}22 \\
21 \\
21\end{array}$ & $\begin{array}{l}194 \\
190 \\
190\end{array}$ & $\begin{array}{l}170 \\
140 \\
160\end{array}$ & $\begin{array}{l}154^{\mathrm{c}} \\
123 \\
110\end{array}$ & $\begin{array}{l}164 \\
144 \\
114\end{array}$ & \\
\hline Standard rock BR & 17.5 & 21 & 190 & 100 & 110 & 114 & \\
\hline $\begin{array}{l}\text { Analysis } 75014 \\
\text { Analysis } 75061\end{array}$ & $\begin{array}{l}14 \\
13\end{array}$ & $\begin{array}{l}48 \\
45\end{array}$ & $\begin{array}{l}1410 \\
1360\end{array}$ & $\begin{array}{l}1160 \\
1050\end{array}$ & $\begin{array}{c}219^{\mathrm{c}} \\
64\end{array}$ & $\begin{array}{l}490 \\
380\end{array}$ & \\
\hline Flanagan (1973) & 9 & 45 & 1350 & 1050 & - & 420 & \\
\hline
\end{tabular}

aAnalyst: J. R. Muysson.

$\mathrm{b}_{\text {Trace. }}$

${ }^{\mathrm{c}}$ Contaminated 
TABLE 2

Loss on Ignition (LOI) ${ }^{\mathrm{a}}$ and Degree of Alteration, Samples Arranged for Each Drill Hole by Increasing Depth

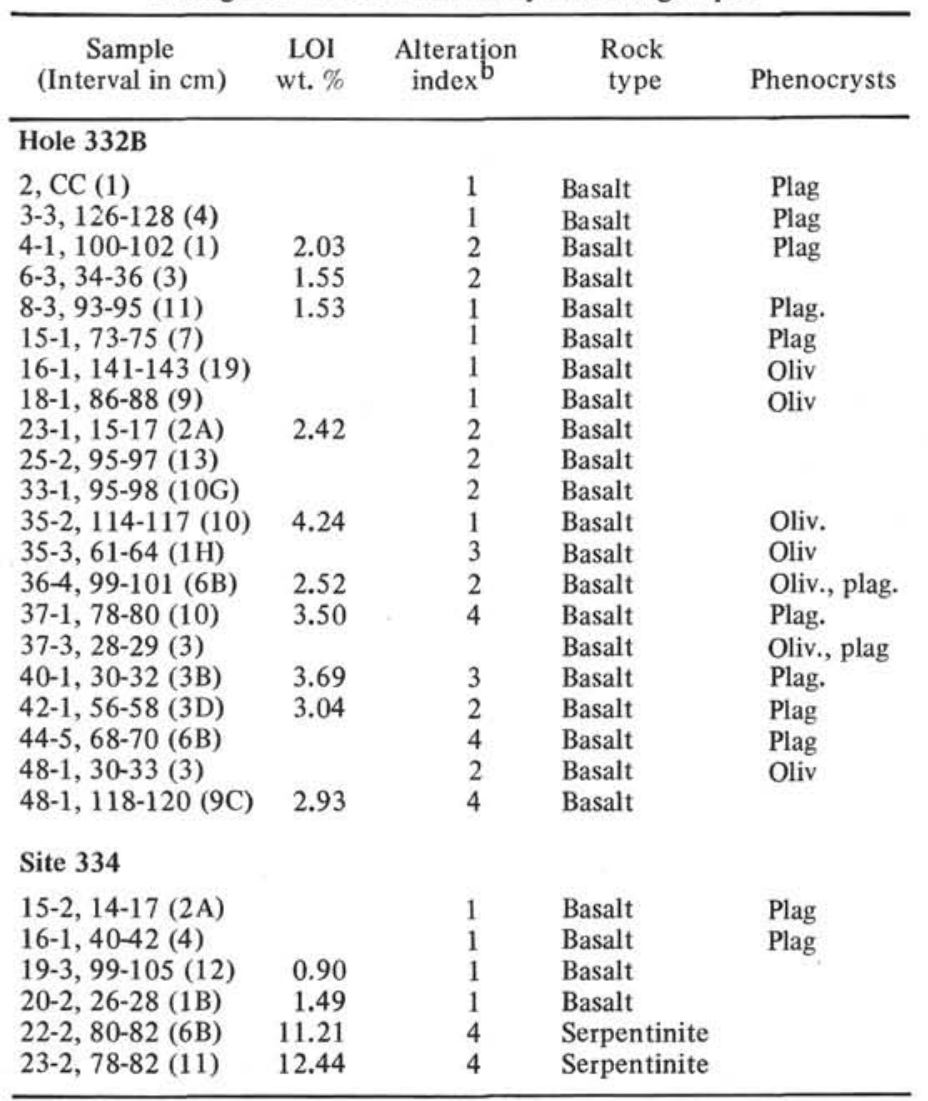

${ }^{\mathrm{a}}$ LOI taken from Dalhousie University compilation of major element analyses dated 26 April 1975.

bAlteration index: 1 least, 4 most altered.

TABLE 3

Mean Values (ppm) of Some Trace Elements in Basic Volcanics

\begin{tabular}{lcccccc}
\hline & $\begin{array}{c}\text { Number of } \\
\text { analyses }\end{array}$ & $\mathrm{Li}$ & $\mathrm{Rb}$ & $\mathrm{Sr}$ & $\mathrm{Ba}$ & $\mathrm{Tl}$ \\
\hline Alkalic basalts & 88 & 9.5 & 45 & 820 & 600 & $0.045^{*}$ \\
Tholeiitic basalts & 139 & 15 & 25 & 280 & 320 & $0.120^{*}$ \\
Spilitic rocks & 152 & 66 & 53 & 280 & 250 & 0.410 \\
Ocean-floor basalts & 50 & 7.5 & 6.2 & 130 & 65 & $0.076^{*}$ \\
Leg 37 basalts & 26 & 8.8 & 5.8 & 104 & 52 & 0.036 \\
\hline
\end{tabular}

Note: All analyses by AAS. Analysts: J. R. Muysson and M. Fratta (values marked ${ }^{*}$ ). 